\title{
Dilation of Time Dilation
}

\author{
Tadeusz Wajda ${ }^{1}$ \\ ${ }^{1}$ Polytechnic University Krakow, Poland \\ Correspondence: Tadeusz Wajda, Polytechnic University Krakow, Poland. E-mail: tadeuszwajda@outlook.com
}

Received: September 29, 2017

Accepted: October 20, 2017

Online Published: November xx, 2017

doi:10.5539/apr.v9n6p67

URL: https://doi.org/10.5539/apr.v9n6p67

\begin{abstract}
The study addresses the issue of the so-called time dilation in the sense of the origin of its creation and the physical existence.

Based on the work of Lorentz, who the lack of displacement of interference fringes in the Michelson interferometer explained wrongly with, shortening one arm of the interferometer, I propose the construction of the light pulse clock, in which to measure the rate of the passage of time is used constancy speed of light in vacuum.

Light clock, the construction of which is described in the paper, stationary in relation to the ether, will measure constant time intervals. The same clock transported, will slow down the pace of his walk as a function of transportation speed $\mathrm{v}$ and that is a novelty, will slow depending on its orientation relative to the direction of motion. Light clock transported transversely with respect to the stationary clock will slow gamma times, transported lengthwise will slow gamma to the second power.

Basing on the obtained dependences I maintain that time dilation defined in the theory of relativity (SR) as the slowing of the lapse of time, does not physically exist and identification the varying pace of walk clock with the pace of lapse of time I consider a fundamental error resulting from the postulates of this theory.
\end{abstract}

\section{Introduction}

In 1881, Mr. Michelson (Michelson, 1881; Michelson \& Morley, 1887) constructed an interferometer, whose aim was to demonstrate the existence of ether or medium in which and against which, propagates light.

Interferometer consisted of two mutually perpendicular arms with a length of about $1.2 \mathrm{~m}$ each, at the ends of whom where mounted mirrors. Two light rays derived from the same source, distributed on a semi-permeable mirror, ran in these two optical systems back and forth and interfered with each other on that separating them before mirror.

Michelson foresaw that if the ether exists and a terrestrial globe turns in it, it should, obtain the movement of bands originated from the interference of these two waveforms. Assuming that if "ether wind" blowing perpendicular to one arm interferometer, that the wind has to blow parallel to the other. After turning the device by 90 degrees, arms somehow turns into functions. Longitudinal frame becomes transverse and transverse - longitudinal.

Unfortunately, the measurements did not show noticeable movements interference fringes. Later measurements made by Michelson and Michelson (1887) with the participation of Morley in 1887 for the interferometer with arm lengths of about $11 \mathrm{~m}$, also the zero offsets results confirmed.

Then scientists intensively searched for lack of those movements.

Then Mr. Lorentz who interpreted this phenomenon as occurring in a stationary ether, analyzed the layout and calculated the times of passage of the light rays in the two mutually perpendicular arms (Lorentz ether theory, 2017). These times he found different. Time of ray flight in the arm "longitudinal" was for him longer and the transverse section - shorter.

Lorentz divided these times by themselves and as a result of division has acquired one of the best known patterns later on so-called "Beta" or also "Lorentz factor". Or "gamma", algebraic inverse of the Beta. This pattern has the form:

$$
\text { gamma }=1 /\left[\left(1-(\mathrm{v} / \mathrm{c})^{\wedge} 2\right]^{\wedge} 0.5\right.
$$

Lorentz submitted a new hypothesis that if the length of the longitudinal arm of the interferometer is divided by this factor, it will shorten the frame due to this time of flight of light also will shorten so, then the time waveforms 
of light in both arms will be identical. It explained the no transfers interference fringes in the Michelson interferometer. And thus "invented" shortening of the length of the segment being in motion with respect to the ether. This shortening is known under the name of the Lorentz contraction.

Surprisingly, this elucidation however erroneous, in the absence of other explanations, has been approved by many contemporary Lorentz scholars.

A dozen years later, in 1905, Mr. Einstein, pretending not heard of Michelson or Lorentz, $\beta$ published work commonly known under the name of "Special Relativity" (SR) (Einstein, 1905; Wielka encyklopedia powszechna PWN, 2017; Wydawnictwo Naukowe PWN, 2017). Works based on his postulate of constancy of the velocity of light 'in a vacuum' and the independence of this velocity of speed of light source. Asked in relation to which the speed of light in a vacuum has a constant value, replied that light has a constant speed in a vacuum in relation "to everything."

In this statement lies the contradiction, because in accordance with the content of the postulate, a source of light in motion, here is the exception. In my opinion, speed of light in vacuum designated in relation to the movable light source is different from the value of $\mathrm{c}$.

Based on these postulates Einstein "guessed" pattern on gamma, invented relativistic time dilation and length contraction. Of course, the coefficient slowing down the lapse of time had identical to the Lorentz form, only that Einstein called it a beta $(\beta)$, whose reversal was the same as the gamma $(\gamma)$.

Many years later (2014) the author of this paper proposed a thesis, the atmospheric air is a stationary medium, form of the ether in relation to whose speed of light is constant in all directions.

It follows a conclusion that the experience of the Michelson type, carried out in still air, in a dark basement, under pressure close to atmospheric pressure at sea level, and did not have right to demonstrate any difference in the speed of light in the air, which depends on the direction of propagation of light.

Therefore, with respect to the ambient air, the interferometer arms remain stationary. When we carry it in the air, or put an instrument in a wind tunnel, then anisotropy of light velocity and displacement of interference fringes will appear.

They will be also present in interferometer working in the air under reduced pressure, which in the years 1905 1923 provided evidence Mr. Dayton Miller performing identical measurements to Michelson at the top of Mt Wilson in California (Miller, 1925).

Mr. Einstein uncritically extrapolated a constancy speed of light in air, to vacuum and ignored measurements of Miller, which showed a non-zero offset values.

\section{The Light Pulse Clock}

By way of construction of the Michelson interferometer we can build a light pulse clock.

Principle of operation and construction of this clock is relatively simple. The clock consists of a pulsed light source and a mirror placed at a distance $\mathrm{L}$ from it, on which emitted pulse of light is reflected and returns to the source. The time interval of flight of the light back and forth, in a vacuum, can be a very good standard unit of time.

In this context, I understand the material or non-material medium filling the space in relation to whose light propagates in all directions at a constant speed $\mathrm{c}$. Each medium has electromagnetic parameters; $\mu$ and $\varepsilon$, and the speed of light is dependent on them.

The medium for this light is of course also vacuum. Because it has the smallest known in nature numeric values $\mu$ and $\varepsilon$, therefore compared with other centers, speed of light in a vacuum has the greatest value.

The mathematical form of this function for electromagnetic waves foresaw Mr. Maxwell, before Hertz experimentally confirmed the existence of electromagnetic waves. It has the form $c=\sqrt{1 / \mu \varepsilon}$ (axwell's equations, 2017).

\section{Schematic design and operation of the light pulse clock}

Light clock consists of rigid rail at one end is installed perpendicular mirror and the other end equipped with a pulsating light source and a light detector to measure the time of flight of the light beam back and forth. Rail, which is the base of this clock has a constant length $\mathrm{L}$.

Schematic design light clock I present in Figure 1 


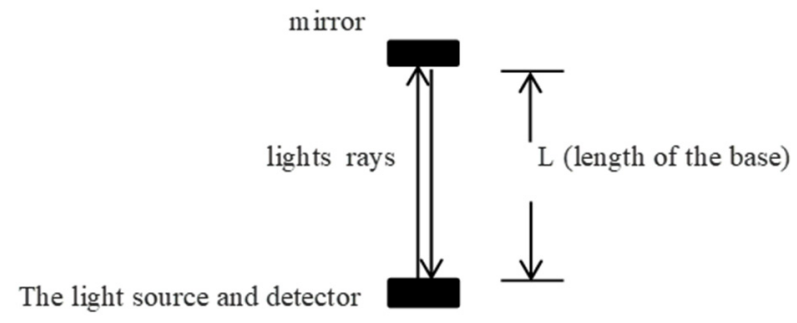

Figure 1

This clock, stationary in relation to the media, will be walked constant pace, depending only on the length $\mathrm{L}$ of the base and value of velocity of light $\mathrm{c}$ in the medium.

I maintain the same clock which is in relation to the medium in motion, will slow down the pace of his walk otherwise will measure the longer time intervals.

Below I show that light clocks, moving, will be to slow down the pace of their walk not only in dependence on the speed $\mathrm{v}$, but also on their orientation relative to the direction of their movement in medium.

\section{Analysis Work of the Stationary Clock}

The source sends short light pulse (Figure 1). Spherical wave propagates in all directions with the same speed equal to c. One of the rays of the wave reaches the mirror, from which reflected returns to the place from which emitted. Time T0, of his flight back and forth, is recorded with photodiode installed near to the source of light.

Time T0 interval that will be measured by this clock, will be 'real time' or 'proper time' that will be stationary and an independent of the orientation of the clock. It will be equal:

$$
\mathrm{TO}=\mathbf{2 L} / \mathrm{c}
$$

Where L - length of the base [m], c - the speed of light $300000 \mathrm{~km} / \mathrm{s}$.

If base (we assume) will have a length $\mathrm{L}=150000 \mathrm{~km}$, this clock will measure 1 second intervals time. $\mathrm{T} 0=1 \mathrm{sec}$. we can build a clock with a much shorter base eg. $\mathrm{L}=1.5 \mathrm{~m}$. The clock will then ticked time intervals equal: $\mathrm{T} 0=2 \mathrm{~L} / \mathrm{c}=2 * 1.5 \mathrm{~m} / 300,000,000 \mathrm{~m} / \mathrm{s}=0.00000001 \mathrm{sec}$ or $=1 \mathrm{e}-8 \mathrm{sec}=10 \mathrm{~ns}$. This time is to be understood as a time interval of his single "tick".

This clock, we can modify. If instead of a single light pulse generator, we will use similar generator but triggered when the previous pulse returns to the detector and equipped with a pulse counter, counting to a hundred million, we can get to the exit clock, also 1-second intervals, of the "real" time T0. It is real time, because "working" light beam flying from the source to the mirror and back, in a stationary clock runs at the shortest possible way. Light ray will fall onto the mirror and reflect from it exactly at right angles. Such a clock, stationary, will then have the fastest possible pace of walk T0.

\section{Analysis of the light clock in motion, in terms of its orientation relative to the direction of his movement.}

\subsection{Clock transported transversely}

We set the clock transversely to the direction of flight of the rocket. Of course, the clock must be installed on the outside of the rocket. Angle $\phi$ between the base clock and the direction of its movement is equal to $\phi=90^{\circ}$. The rocket moves at a speed $\mathrm{v}$ defined as the ratio of the traveled distance divided by the stationary time.

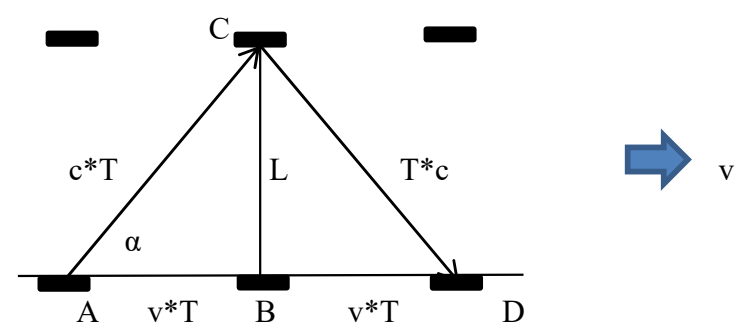

Figure 2 
Figure 2 will be helpful in calculating the walking speed of this clock in motion. Pace of walking of this clock Tt, will be function of speed $\mathrm{v}$, its movement with respect to the medium in which light propagates at a constant speed c.

The light pulse emitted by the generator at the point $\mathrm{A}$, runs along the hypotenuse of a right triangle $\mathrm{ABC}$. In the interval $\mathrm{T}=1 / 2 \mathrm{Tt}$ clock will travel the way $\mathrm{AB}$ and at the same time, the light pulse will pass the way $\mathrm{AC}$.

We see that the light beam runs at an angle $\boldsymbol{\alpha}$ to the direction of movement of the clock and comes to the detector, which will find at point $\mathrm{D}$ moving with speed $\mathrm{v}$.

Considering geometry of flight (triangle $\mathrm{ABC}$ ) and applying the Pythagorean theorem, we formulate the equation:

$$
(0.5 \mathrm{Tt} * \mathrm{c}) \wedge 2=(0.5 \mathrm{Tt} * \mathrm{v}) \wedge 2+\mathrm{L}^{\wedge} 2
$$

From this we get the flight time of light pulse from the generator (point A) to the mirror (point B) and back to the detector, which finds at point $\mathrm{D}$.

This clock will measure time intervals, let's call them transverse, with time interval Tt, equal:

$$
\mathrm{Tt}=2 \mathrm{~L} /\left(\mathrm{c}^{\wedge} \mathbf{2}-\mathrm{v}^{\wedge} \mathbf{2}\right)^{\wedge} \mathbf{0 . 5}
$$

This clock will walk in relation to a stationary clock slower, because "working" light beam will run along a zigzag line that is along hypotenuses formed by way of light Lc, way vT of clock and constant length $\mathrm{L}$ of base (Figure 2).

\subsection{Clock moving lengthwise.}

The same clock now we set in parallel to the direction of movement. Light pulse emitted from the source (pulse generator) runs to the mirror, which gets away from him in the direction of movement at a speed v. This ray is reflected from mirror and returns to the detector, which "is approaching". This running away and approach are to be understood as a phenomenon of movement in relation to a stationary medium in which light propagates at a constant speed c.

Figure 3 facilitates way of calculation of the walking speed of the clock working as "longitudinal".

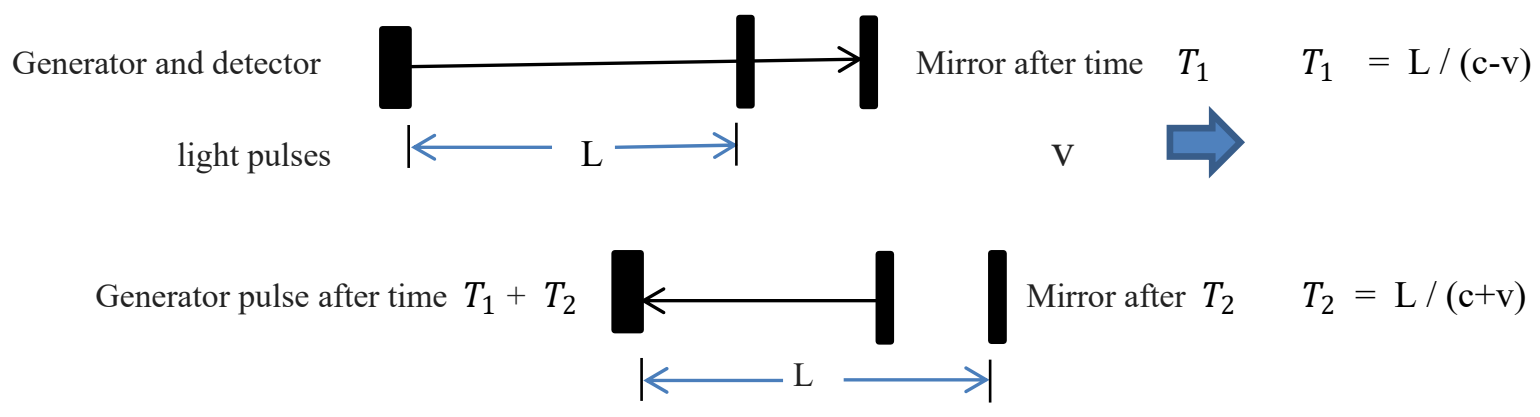

Figure 3

Time $\mathrm{Tl}$ of pulse flight back and forth will be equal to the sum of the time intervals $\mathrm{T} 1$ and $\mathrm{T} 2$.

Clock moving lengthwise, will measure time interval equal

$$
\mathrm{Tl}=\mathrm{L} /(\mathrm{c}+\mathrm{v})+\mathrm{L} /(\mathrm{c}-\mathrm{v}) .
$$

After algebraic transformation, this time interval will be equal to:

$$
\mathbf{T l}=\mathbf{2 L c} /\left(\mathbf{c}^{\wedge} \mathbf{2}-\mathbf{v}^{\wedge} \mathbf{2}\right)
$$

The calculation of the walking speed of the clock section in relation to the walking speed of a stationary clock.

We count how many times slower compared to the clock stationary will walk clock transported with speed v, transversely to direction of its movement.

We divide walking pace [1] by walking pace [0]

$$
\begin{gathered}
\mathrm{Tt} / \mathrm{T} 0=2 \mathrm{~L} /\left[\mathrm{c}^{\wedge} 2-\mathrm{v}^{\wedge} 2\right]^{\wedge} 0.5 / 2 \mathrm{~L} / \mathrm{c}=1 /\left[1-(\mathrm{v} / \mathrm{c})^{\wedge} 2\right]^{\wedge} 0.5 \\
\text { Time } \mathrm{Tt}=\mathrm{T} 0 * 1 /\left[1-(\mathrm{v} / \mathrm{c})^{\wedge} 2\right]^{\wedge} 0.5
\end{gathered}
$$


We note that the value $1 /\left[1-(\mathrm{v} / \mathrm{c})^{\wedge} 2\right]^{\wedge} 0.5$ is equal to Lorentzian gamma.

$$
\mathrm{Tt}=\mathrm{T} 0 * \text { gamma } \quad \text { where gamma }=1 /\left[1-(\mathrm{v} / \mathrm{c})^{\wedge} 2\right]^{\wedge} 0.5
$$

Note: The calculation is more convenient if to apply the formula derived by me, based on trigonometric functions.

Since the hypotenuse of the triangle $\mathrm{ABC}$ (Figure 2) is equal to $\mathrm{L}=\mathrm{c} * \mathrm{~T} 0$ and the hypotenuse of the triangle is equal $\mathrm{c} * \mathrm{~T}$, so the ratio of these sides cT0 / cT is sine of angle $\alpha$. After simplifying by the $\mathrm{c}$ receive $\mathrm{T} 0 / \mathrm{T}=\sin \alpha$.

$$
\mathrm{v} * \mathrm{~T} / \mathrm{T} * \mathrm{c}=\cos \alpha
$$

Angle $\alpha=\operatorname{arc} \cos (\mathrm{v} / \mathrm{c})$

From this time $\mathrm{T}=\mathrm{T} 0 / \sin \operatorname{arc} \cos (\mathrm{v} / \mathrm{c})$.

According to conventional terminology, gamma $=1 / \sin \arccos (\mathbf{v} / \mathbf{c})$.

An interesting letter time interval $\mathrm{T}$ is equal to $\mathrm{T}=\mathrm{T} 0$ *gamma.

To derive this formula for gamma, you do not need any new assumptions or postulates; classical laws of motion, optics and knowledge of trigonometric functions are sufficient.

The pattern is the same as the classic relativistic pattern,

\section{The calculation of the walking speed of the longitudinal clock}

$$
\operatorname{gamma}=1 / \sin \operatorname{arc} \cos (v / c)==1 /[1-(v / c) \wedge 2]^{\wedge} 0.5
$$

We count how many times slower than a stationary clock, the clock will walk when is transported with a speed $\mathrm{v}$, lengthwise that is in parallel to the direction of its movement.

Divide walking pace [2] by [0]

$$
\mathrm{Tl} / \mathrm{T} 0=2 \mathrm{Lc} /\left[\mathrm{c}^{\wedge} 2-\mathrm{v}^{\wedge} 2\right] / 2 \mathrm{~L} / \mathrm{c}=1 /\left[1-(\mathrm{v} / \mathrm{c})^{\wedge} 2\right]
$$

We note that the ratio of the times is gamma to the second power

The time $\mathrm{Tl}=\mathrm{T} 0 * 1 /\left[1-(\mathrm{v} / \mathrm{c})^{\wedge} 2\right]$

We have, $\mathbf{T l}=\mathbf{T} \mathbf{0} * \operatorname{gamma}^{\wedge} \mathbf{2}$

In result from the above analysis and calculations that:

Light clock moving and oriented transversely, will slow the pace of his walk in relation to a stationary clock, in accordance with Lorentz, gamma times.

The same clock oriented lengthwise, will slow the pace of his walk, gamma to the second power times!

The concept of "walking pace" should be understood as measured out with this clock time interval T, which compared with interval T0 which is measures with the stationary clock - will always be longer.

From the above it follows that every light clock in motion - will slow down. The rate of slowing down will be a function of speed $\mathrm{v}$ and the orientation of the clock in relation to the direction of his movement.

\section{A few comments criticisms of the currently valid Special Theory of Relativity}

Mr. Einstein is the author or inventor of "time dilation". He noticed that the clock transported, moving in vacuum with a speed $\mathrm{v}$, it is the same clock, the clock with identical structure like a stationary clock.

His reasoning was the following. Since the same clock, identically constructed, transported with a speed v, slowing down his walk gamma times, well, according to Mr. Einstein, there is no other explanation of this phenomenon, as such, the time in the system of reference in motion, has to slow down the pace of his lapse otherwise must undergo dilation ...

I think the greater nonsense it's even hard to imagine.

\section{Argumentation}

Light pulse clock, despite identical mechanical design, during its movement, behaves and works significantly different from stationary clock.

In the stationary clock, "working" light beam runs along the shortest possible way, because runs to the mirror at right angles and is reflected from it at the same right angle. So it comes the mirror and returns to the source, along the shortest possible, straight line. Due to this pace of his walk is maximum. Stationary clock will measure the shortest possible time intervals. 
The clock transported transversely to the direction of motion, working light ray, runs to the mirror at an angle $\alpha$, (as defined in relation to the direction of travel and mirror plane). The value of this angle is equal to

$$
\alpha=\arccos (\mathrm{v} / \mathrm{c}) \text {. }
$$

At the same angle $\alpha$, this ray is reflected from the mirror, but does not return to the point in space from which it was generated. He arrives at the site, which will house the generator (and the detector coupled with), which during the flight of working ray covered distance, $\mathrm{s}=\mathrm{v} * \mathrm{~T} 0$.

Unfortunately, the "brilliant" conclusion of Mr. Einstein, it's not the clock slows down his walk, but time slows down his lapse, was by many scientists and world press interpreted as the greatest scientific achievement of the twentieth century.

Especially, with this slowing the lapse of time by Mr. Einstein, can be so large, that this elapse can be stopped. It just suffices to move enough fast. And you can even go back in time, if would manage to exceed the speed of light!

And many contemporaries Einstein scientists believed into those nonsense. And many of our contemporaries scientists still believe in these absurdities.

\section{Summary}

I proved that the light clock transported transversely to the direction of travel, in relation to the stationary clock slows down his walk gamma times.

I proved that the clock oriented lengthwise to the direction of travel, in relation to the clock transported transversely, slows down his walk again gamma times.

I showed that the light clock oriented lengthwise, in relation to the stationary clock, slows down his walk gamma* gamma times.

These calculations proved that the light clocks slow down their walk depending not only on the transportation speed $\mathrm{v}$, but also depending on their orientation relative to the direction of their movement.

The differences are large; clock oriented transversely slows the pace according with gamma function, while the same clock oriented longitudinally, slows down his walk gamma to the second power.

I stress, the above coefficients gamma determine the walking pace of light clock and not slowing down the passage of time. Time is a material constant therefore his pace elapse can't change.

In the entire Universe time elapses in its constant invariant pace.

Identifying the walking pace of clocks, including "atomic", with the pace of the passage of time, I consider the greatest scientific scam of the twentieth century.

\section{Concluding remarks.}

For a comparison of the differences between values of normal and square dilation, we assume missile velocity $\mathrm{v}=$ $0.1 \mathrm{c}$. Then gamma "normal" that is specified for the transverse clock, will be equal to 1,005 , while gamma "square", or longitudinal, will have a value of $1,005^{\wedge} 2=1.01$.

For speed $\mathrm{v}=0.999 \mathrm{c}$, the corresponding gamma values will be equal 22.35 and 500.25.

An inquisitive reader will notice that the light clock shown in Figure 1, which is stationary, in the real space intergalactic vacuum which we of Earth orbiting the Sun as it orbits around the nucleus of the Milky Way, which in turn wanders somewhere to the south of the visible Universe, it this clock also will not order the correct rate of the passage of time, which I called a real time or stationary. And the reader will be right.

The use of the light clock will be equally as the existence of gamma square - unexpected.

With the help of such a clock placed in a vacuum, we can determine our direction, in the sense of US hiking in the universe. Just in relation to a number of distant pulsars, or galaxies, which can be treated as cosmic lighthouses, we can find the direction in which our clock will walk slowest. This will mean that in this direction it moves. Based on the indications of our clock, we can also determine its speed in absolute intergalactic vacuum.

Speed is defined in relation to the Universal Reference System (URS), which is the visible Universe. For this purpose we use the clock longitudinal. Knowing the gamma value defined as the ratio of the walk paces of longitudinal clock to a transverse one, or classical Lorentz gamma, we can determine its speed of movement in relation to the fixed, three-dimensional space, characterized by that in it every motion of material bodies takes place and the light propagates with the constant speed c. Space shall be defined as a Universal Reference System. What my "discovery" may have consequences for the theory of relativity? 
So far we did not include the possibility of other than a specific by Lorentz, the rate of slowing down of clock pace. The rate defined by a specific coefficient gamma. While this rate is misidentified with the rate of the passage of time.

Meanwhile, the clock in the same inertial system, which my URS is transported with the same speed of $v$, but oriented parallel to the direction of travel, will slow down significantly more!

Physicists faithful to demands and theses of special theory relativity, following its author argue that the clocks always walk in the same pace only time undergoes dilation, which is evident absurdity.

We assume for the moment that this is true.

My discovery created a problem, which dilation will be now applied in SR? The transverse or longitudinal. Transverse, used since the times of the Lorentz to the present, or longitudinal, which in my humble opinion, should be used in all systems where previously erroneously applied transverse dilation.

It will be an inexhaustible source of new experiences and new industrial themes for academic discussion. In this new absurdities connected with the theory of relativity.

How will behave, which is what will notice an imaginary observer, who with his frame of reference, by the great telescopes, will observe the walking speed of two clocks in the rocket, one of which will be positioned transversely to the direction of flight, and the second longitudinally? It is found that, however, this "time dilation" is a real phenomenon, possible to observe for observers by going along with the clocks in their frame reference. Thus, relativistic jump from one system to another to see it - it is not necessary at all.

Has time dilation, as according to Mr. Einstein - light a dual character?

And how will grow older this cult paradox twin since the aging process will depend on this, which clock will be observed? And if by chance it will not age faster in the flight direction of a significantly more slowly in the direction transverse to the direction of flight of the rocket?

And what about the process of the relativistic contraction, along with the rocket course? In a direction transverse to the direction of flight - to the left and owing to turn - more?

Perhaps for the faithful believer SR my calculations will prove illegal in the sense of compliance with the postulate of constancy of the velocity of light and the relativity of motion. In the derivation of formula for the walking pace of longitudinal clock model of Lorentz and basing on the principles of classical physics, I relied on the constancy of the velocity of light in vacuum and on speed of clock motion also in a vacuum and not in relation to some undefined observer or also another reference system to which it is necessary to jump to the "time dilation" see.

And that's why I came out with "time dilation of dilation".

What should be understood that the light clock transported lengthwise in relation to the fixed clock, slows down his pace according to the gamma function to the second power.

Will not it be outright beating source of new absurdities affectionately called paradoxes of this absurd "theory"?

Because so far by any applicable rules in theory, twin will be shortened only in the direction of travel. And here comes that he will become thinner also in the transverse direction.

For small speed difference will be negligibly small but for examples of $v=0.999 \mathrm{c}$, already enormous. Pilot becomes cross thinner 22 times but will reduce his thickness the 500 times. And the speed v $=0.9999 \mathrm{c}$ is it just a sheet of thick row $1 / 5000$ thickness astronaut at the waist, when a rocket was going to start. In the same proportion the dimensions of the rocket will decrease.

With flight speed equal to $\mathrm{c}$, rocket would have been annihilated?

\section{Acknowledgements}

Translation into English and adjustment, PhD Janusz Maczka, Poland.

\section{References}

Einstein, A. (1905). Zur elektrodynamik bewegter körper. Annalen der physik, 322(10), 891-921.

Lorentz ether theory. (2017). In Wikipedia, the free encyclopedia. Retrieved from https://en.wikipedia.org/ wiki/Lorentz_ether_theory

Lorentz, H. A. (1904). Electromagnetic phenomena in a system moving with any velocity smaller than that of light. Proceedings of the Royal Netherlands Academy of Arts and Sciences, 6, 809-831 
Maxwell's equations. (2017). In Wikipedia, the free encyclopedia. Retrieved from https://en.wikipedia.org/wiki/Maxwell\%27s_equations

Michelson, A. A. (1881). The relative motion of the Earth and of the luminiferous ether. American Journal of Science, (128), 120-129. https://doi.org/10.2475/ajs.s3-22.128.120

Michelson, A. A., \& Morley, E. W. (1887). On the Relative Motion of the Earth and the Luminiferous Ether. American Journal of Science, 34, 333-345. https://doi.org/10.2475/ajs.s3-34.203.333

Miller, D. C. (1925). Ether-drift experiments at Mount Wilson. Proceedings of the National Academy of Sciences, 11(6), 306-314.

Miller, D. C. (1933). The ether-drift experiment and the determination of the absolute motion of the earth. Reviews of modern physics, 5(3), 203. Retrieved from http://www.orgonelab.org/EtherDrift/MillerRMP1933.pdf

Oostra, B. (2012). Measurement of the Earth's rotational speed via Doppler shift of solar absorption lines. American Journal of Physics, 80(5), 363-366.

Sagnac, G. (1913). L'éther lumineux démontré par l'effet du vent relatif d'éther dans un interféromètre en rotation uniforme. CR Acad. Sci., 157, 708-710.

Voigt, W. (1887). Ueber das Doppler'sche Princip. Göttinger Nachrichten, (7), 41-51 Reprinted with additional comments by Voigt in Physikalische Zeitschrift, XVI, 381-386 (1915).

Wielka encyklopedia powszechna PWN. (2017). In Wikipedia, the free encyclopedia. Retrieved from https://pl.wikipedia.org/wiki/Wielka_encyklopedia_powszechna_PWN

Wydawnictwo Naukowe PWN. (2017). In Wikipedia, the free encyclopedia. Retrieved from https://pl.wikipedia.org/wiki/Wydawnictwo_Naukowe_PWN

\section{Copyrights}

Copyright for this article is retained by the author(s), with first publication rights granted to the journal.

This is an open-access article distributed under the terms and conditions of the Creative Commons Attribution license (http://creativecommons.org/licenses/by/4.0/). 